\title{
Dynamic of Non-Autonomous Vector Infectious Disease Model with Cross Infection
}

\author{
Weiwei Ji*, Shenghua Zou, Jishang Liu, Quanben Sun, Lijun Xia \\ Department of Basic Course Education, Ji'an College, Ji'an, China \\ Email: ‘923660819@qq.com
}

How to cite this paper: Ji, W.W., Zou, S.H., Liu, J.S., Sun, Q.B. and Xia, L.J. (2020) Dynamic of Non-Autonomous Vector Infectious Disease Model with Cross Infection. American Journal of Computational Mathematics, 10, 591-602. https://doi.org/10.4236/ajcm.2020.104034

Received: November 20, 2020

Accepted: December 18, 2020

Published: December 21, 2020

Copyright $\odot 2020$ by author(s) and Scientific Research Publishing Inc. This work is licensed under the Creative Commons Attribution International License (CC BY 4.0).

http://creativecommons.org/licenses/by/4.0/ (c) (i) Open Access

\begin{abstract}
In the article, we established a non-autonomous vector infectious disease model, studied the long-term dynamic behavior of the system, and obtained sufficient conditions for the extinction and persistence of infectious diseases by constructing integral functions.
\end{abstract}

\section{Keywords}

Non-Autonomous, Seasonal Variability, Vector Infectious Disease

\section{Introduction}

In real life, we are often confused by infectious diseases. Infectious diseases include humans, animals, plant infectious diseases, especially human infectious diseases, such as tuberculosis, AIDS/HIV, malaria, which are the top three single disease killers of health in the world. According to the World Health Organization statistics, in 2002 about 70 million people are infected with AIDS, causing around 20 million deaths. In recent years, each year more than 560 million people infected with AIDS [1] [2]. The control of infectious diseases spread has aroused great interest of the people and many mathematical models are established (see [3] [4] [5] [6]) to understand the mechanism of disease transmission, and to prevent or slow down the transmission of infectious diseases. In order to effectively control the spread of infectious diseases, we often introduce three control strategies in the model: cohort immunization, time-dependent pulse vaccination, and state-dependent vaccination. The first strategy details a continuous vaccination effort of susceptible individuals, while the second and third strategies involve vaccinating a significant fraction of the susceptible population in a short period of time [7].

In recent years, some mathematical models incorporating treatment have been established and investigated by many researchers [8]-[15]. Infectious dis- 
eases are the most important biosecurity issues, and every country should pay attention and strive to have a maximal capacity treatment for diseases. Therefore, it is vital to describe the limited capacity for treatment [16]. In [12], Wang and Ruan proposed the constant treatment function of diseases in an SIR epidemic model. According to this model when people get sick and must be hospitalized but there are limited beds in hospitals, or there is not enough medicine for treatments, should be considered and simulated the limited resources for the treatment of patients. Wang [13] researched the piecewise linear treatment function. The model is assumed that treatment rate is proportional to the number of infectives below the capacity and is a constant when the number of infectives is greater than the capacity. In [13], Wang adopted a constant treatment, which is suitable for the case of a large number of infectives. Zhang and Liu [17] introduced a continuously differentiable treatment function, which describes the saturation phenomenon of the limited medical resources. Zhang and Kang [15] proposed discontinuous treatment function in an SEIR epidemic model, which describes that the treatment rate has at most a finite number of jump discontinuities in every compact interval.

Some infectious diseases are transmitted by vector, such as Malaria, Dengue and West Nile virus, which spread by Mosquitoes. The maintenance and resurgence of vector-borne diseases are related to ecological changes that favor increased vector densities or vector-host interactions, among other factors [18]. However, travel and transport are a major factor in the spread of vector-borne diseases, we have reasons to believe that the spatial movement of humans may be important for the epidemiology of vector-borne diseases. Every year there are more than 1 billion cases and over 1 million deaths from vector-borne diseases such as malaria, dengue, schistosomiasis, human African try-panosomiasis, leishmaniasis, Chagas disease, yellow fever, Japanese encephalitis and onchocerciasis [19]. So the vector-borne is a very important part of the transmission of epidemic diseases.

The structure of this paper is organized as follows. Section 2 presents the vector-borne diseases model. And positivity and boundary of the model (1.1) are studied. In Section 3 and 4, we deal with the existence and permanence of model (1.1). In Section 5, we had a brief discussion.

\section{Definitions and Preliminaries}

Based on [20], we get the following vector infectious disease model:

$$
\left\{\begin{array}{l}
\frac{\mathrm{d} S(t)}{\mathrm{d} t}=\Lambda(t)-\beta_{1}(t) S(t) I(t)-\beta_{2}(t) S(t) Y(t)-\mu(t) S(t), \\
\frac{\mathrm{d} I(t)}{\mathrm{d} t}=\beta_{1}(t) S(t) I(t)+\beta_{2}(t) S(t) Y(t)-\mu(t) I(t), \\
\frac{\mathrm{d} X(t)}{\mathrm{d} t}=L(t)-\gamma(t) I(t) X(t)-\mu_{v}(t) X(t), \\
\frac{\mathrm{d} Y(t)}{\mathrm{d} t}=\gamma(t) I(t) X(t)-\mu_{v}(t) Y(t) .
\end{array}\right.
$$


with initial value

$$
S(0)>0, I(0)>0, X(0)>0, Y(0)>0
$$

where the variables $S(t), I(t), X(t)$ and $Y(t)$ represent susceptible host, infected host, susceptible vector and infection vector, respectively. $\Lambda(t)$ represents the input rate of susceptible hosts, $\beta_{i}(t)(i=1,2)$ means effective contact rate. $\mu(t)$ and $\mu_{v}(t)$ represents the natural mortality of the host and the vector, respectively. $L(t)$ represents the birth rate of the newborn vectors, A represents the effective bite rate of the vector.

\section{Assumption 2.1}

1) Functions $\Lambda(t), \beta_{1}(t), \beta_{2}(t), \mu(t), \gamma(t)$ and $\mu_{v}(t)$ are positive, bounded and continuous on $[0,+\infty)$.

2) There exist constants $\omega_{i}>0(i=1,2,3,4)$ such that

$$
\begin{gathered}
\int_{t}^{t+\omega_{1}} \Lambda(s) \mathrm{d} s>0, \liminf _{t \rightarrow+\infty} \int_{t}^{t+\omega_{2}} \beta_{1}(s) \mathrm{d} s>0, \\
\liminf _{t \rightarrow+\infty} \int_{t}^{t+\omega_{3}} \beta_{2}(s) \mathrm{d} s>0, \liminf _{t \rightarrow+\infty} \int_{t}^{t+\omega_{4}} \mu(s) \mathrm{d} s>0 .
\end{gathered}
$$

In what follows, we denote $N(t)=S(t)+I(t), N_{v}(t)=X(t)+Y(t)$ and $N(t)$ the solution of

$$
\frac{\mathrm{d} N(t)}{\mathrm{d} t}=\Lambda(t)-\mu(t) N(t)
$$

$N_{v}(t)$ the solution of

$$
\frac{\mathrm{d} N_{v}(t)}{\mathrm{d} t}=L(t)-\mu_{v}(t) N_{v}(t)
$$

with initial value $S(0)>0, S(0)>0, N(0)=S(0)+I(0)>0$, $N_{v}(0)=X(0)+Y(0)>0$.

\section{Proposition 2.2}

1) There exist constants $m_{1}>0$ and $M_{1}>0$, which are independent from the chioce of initial value $N(0)>0$, such that

$$
0<m_{1} \leq \liminf _{t \rightarrow+\infty} N(t) \leq \limsup _{t \rightarrow+\infty} N(t) \leq M_{1}<+\infty .
$$

2) There exist constants $m_{2}>0$ and $M_{2}>0$, which are independent from the chioce of initial value $N_{v}(0)>0$, such that

$$
0<m_{2} \leq \liminf _{t \rightarrow+\infty} N_{v}(t) \leq \limsup _{t \rightarrow+\infty} N_{v}(t) \leq M_{2}<+\infty \text {. }
$$

3) The solution $(S(t), I(t), X(t), Y(t))$ of system (1.1) with initial value (2.2) exists, uniformly bounded and

$$
S(t)>0, I(t)>0, X(t)>0, Y(t)>0,
$$

for all $t>0$.

For $p>0, q>0$ and $t>0$ we define

$$
G(p, t)=\left[\beta_{1}(t)+p \beta_{2}(t)\right] N(t)-\mu(t)+\mu_{v}(t)
$$

and 


$$
W(p, t)=p I(t)-Y(t),
$$

where $I(t)$ and $Y(t)$ are solutions of system (1.1). In Sections 3 and 4 we use the following lemma in order to investigate the longtime behavior of system (2.1).

Lemma 2.3 If there exist positive contants $p>0$ and $T_{1}>0$ such that $G(p, t)<0$ for all $t \geq T_{1}$, then there exists $T_{2} \geq T_{1}$ such that either $W(p, t)>0$ for all $t \geq T_{2}$ or $W(p, t) \leq 0$ for all $t \geq T_{2}$.

Proof. Suppose that there does not exist $T_{2} \geq T_{1}$ such that $W(p, t)>0$ for all $t \geq T_{2}$ or $W(p, t) \leq 0$ for all $t \geq T_{2}$ hold. Then there necessarily exists $s_{1} \geq T_{1}$ such that $W\left(p, s_{1}\right)=0$ and $\left.\frac{\mathrm{d} W(p, t)}{\mathrm{d} t}\right|_{t=s_{1}}>0$. Hence we have

$$
p I\left(s_{1}\right)=Y\left(s_{1}\right)
$$

and

$$
\begin{aligned}
& p\left[\beta_{1}\left(s_{1}\right) S\left(s_{1}\right) I\left(s_{1}\right)+\beta_{2}\left(s_{1}\right) S\left(s_{1}\right) Y\left(s_{1}\right)-\mu\left(s_{1}\right) I\right]\left(s_{1}\right) \\
& -\gamma\left(s_{1}\right) I\left(s_{1}\right) X\left(s_{1}\right)+\mu_{v}\left(s_{1}\right) Y\left(s_{1}\right) \\
& =p I\left(s_{1}\right)\left[\beta_{1}\left(s_{1}\right) S\left(s_{1}\right)-\mu\left(s_{1}\right)\right]+Y\left(s_{1}\right)\left[p \beta_{2}\left(s_{1}\right) S\left(s_{1}\right)+\mu_{v}\left(s_{1}\right)\right] \\
& \quad-\gamma\left(s_{1}\right) I\left(s_{1}\right) X\left(s_{1}\right) \\
& >0
\end{aligned}
$$

Substituting (2.8) into (2.9) we have

$$
\begin{aligned}
0< & p I\left(s_{1}\right)\left[\beta_{1}\left(s_{1}\right) S\left(s_{1}\right)-\mu\left(s_{1}\right)+p \beta_{2}\left(s_{1}\right) S\left(s_{1}\right)+\mu_{v}\left(s_{1}\right)\right] \\
& -\gamma\left(s_{1}\right) I\left(s_{1}\right) S_{v}\left(s_{1}\right) \\
\leq & p I\left(s_{1}\right) G\left(p, s_{1}\right) .
\end{aligned}
$$

From 3) of Proposition 2.2, we have $G\left(p, s_{1}\right)>0$, which is a contradiction.

\section{Extinction of Infectious Population}

In this section, we obtain conditions for the extinction of infectious population of system (2.1). The definition of the extinction is as follows:

Definition 3.1. We say that the infectious population of system (2.1) is extinct if

$$
\lim _{t \rightarrow+\infty} I(t)=0, \lim _{t \rightarrow+\infty} Y(t)=0 .
$$

From system (2.1), it's easy to prove that if one of the above equalities hold, then the other one is certainly hold. We give one of the main results of this paper.

Theorem 3.2. If there exist positive constants $\lambda>0, p>0, q>0$ and $T_{1}>0$ such that

$$
\begin{gathered}
R_{1}(\lambda, p) \triangleq \limsup _{t \rightarrow+\infty} \int_{t}^{t+\lambda}\left\{\left(\beta_{1}(s)+p \beta_{1}(s)\right) N(s) p-\mu(s)\right\} \mathrm{d} s<0, \\
R_{1}^{\prime}(\lambda, p) \triangleq \limsup _{t \rightarrow+\infty} \int_{t}^{t+\lambda}\left\{\frac{1}{p} \gamma(s) N_{v}(s)-\mu_{v}(s)\right\} \mathrm{d} s<0,
\end{gathered}
$$


and $G(p, t)<0$ for all $t \geq T_{1}$, then the infectious population of system (2.1) is extinct.

Proof. From Lemma 2.3, we only have to consider the following two cases.

1) $W(p, t)>0$ for all $t \geq T_{2}$.

2) $W(p, t) \leq 0$ for all $t \geq T_{2}$.

First we consider the case 1). From the second equation of system (2.1), we have

$$
\begin{aligned}
\frac{\mathrm{d} I(t)}{\mathrm{d} t} & =\beta_{1}(t) S(t) I(t)+\beta_{2}(t) S(t) Y(t)-\mu(t) I(t) \\
& <\left\{\beta_{1}(t) S(t)+p \beta_{2}(t) S(t)-\mu(t)\right\} I(t) \\
& <\left\{\left(\beta_{1}(t)+p \beta_{1}(t)\right) N(s) p-\mu(t)\right\} I(t) .
\end{aligned}
$$

Hence, we obtain

$$
I(t)<I\left(T_{2}\right) \exp \left(\int_{T_{2}}^{t}\left\{\left(\beta_{1}(s)+p \beta_{1}(s)\right) N(s) p-\mu(s)\right\} \mathrm{d} s\right)
$$

for all $t \geq T_{2}$. From (3.1) we see that there exist constants $\delta_{1}>0$ and $T_{3}>T_{2}$ such that

$$
\int_{t}^{t+\lambda}\left\{\left(\beta_{1}(s)+p \beta_{1}(s)\right) N(s) p-\mu(s)\right\} \mathrm{d} s<-\delta_{1},
$$

for all $t \geq T_{3}$. From (3.3) and (3.4), we have $\lim _{t \rightarrow+\infty} I(t)=0$. Then it follows from $p I(t)>Y(t)$ for all $t \geq T_{2}$ that $\lim _{t \rightarrow+\infty} Y(t)=0$.

Next we consider the case 2). From the fourth equation of system (2.1), we have

$$
\begin{aligned}
\frac{\mathrm{d} Y(t)}{\mathrm{d} t} & =\gamma(t) I(t) X(t)-\mu_{v}(t) I_{v}(t) \\
& \leq \frac{1}{p} \gamma(t) Y(t) X(t)-\mu_{v}(t) Y(t) \\
& <\left(\frac{1}{p} \gamma(t) N_{v}(t)-\mu_{v}(t)\right) Y(t)
\end{aligned}
$$

Hence we have

$$
Y(t)<Y\left(T_{2}\right) \exp \left(\int_{T_{2}}^{t}\left\{\frac{1}{p} \gamma(s) N_{v}(t)-\mu_{v}(s)\right\} \mathrm{d} s\right)
$$

From (3.2) we see that there exist constants $\delta_{2}>0$ and $T_{4}>T_{2}$ such that

$$
\int_{t}^{t+\lambda}\left\{\frac{1}{p} \gamma(s) N_{v}(t)-\mu_{v}(s)\right\} \mathrm{d} s<-\delta_{2},
$$

for all $t \geq T_{4}$. From (3.6) and (3.7), we have $\lim _{t \rightarrow+\infty} Y(t)=0$. Then it follows from $I(t) \leq \frac{1}{p} Y(t)$ for all $t \geq T_{2}$ that $\lim _{t \rightarrow+\infty} I(t)=0$.

\section{Permanence of Infectious Population}

In this section, we get sufficient conditions for the permanence of infectious 
population of system (2.1). The definition of the permanence is as follows:

Definition 4.1. We say that the infectious population of system (2.1) is permanent if there exist positive constants $I_{1} \geq 0$ and $I_{2} \geq 0$, which are independent from the choice of initial value satisfying (2.2), such that

$$
0<I_{1} \leq \liminf _{t \rightarrow+\infty} I(t) \leq \limsup _{t \rightarrow+\infty} I(t) \leq I_{2}<+\infty .
$$

We give one of the main results of this paper.

Theorem 4.2. If there exist positive constants $\lambda>0, p>0, q>0$ and $T_{1}>0$ such that

$$
\begin{gathered}
R_{2}(\lambda, p) \triangleq \liminf _{t \rightarrow+\infty} \int_{t}^{t+\lambda}\left\{\left(\beta_{1}(s)+p \beta_{1}(s)\right) N(s) p-\mu(s)\right\} \mathrm{d} s>0, \\
R_{2}^{\prime}(\lambda, q) \triangleq \liminf _{t \rightarrow+\infty} \int_{t}^{t+\lambda}\left\{\frac{1}{p} \gamma(s) N_{v}(s)-\mu_{v}(s)\right\} \mathrm{d} s>0,
\end{gathered}
$$

and $G(p, t)<0$ for all $t \geq T_{1}$, then the infectious population of system (2.1) is permanent.

Before we give the Proof of Theorem 4.2, we introduce the following lemma.

Lemma 4.3. If there exist positive constants $\lambda>0, p>0$ and $T_{1}>0$ such that (4.1), (4.2) and $G(p, t)<0$ hold for all $t \geq T_{1}$, then $W(p, t)>0$ for all $t \geq T_{2} \geq T_{1}$, where $T_{2}$ is given as in lemma 2.3 .

Proof. From Lemma 2.3 we have only two cases to discuss, $W(p, t)>0$ for all $t \geq T_{2}$ or $W(p, t) \leq 0$ for all $t \geq T_{2}$. Suppose that $W(p, t) \leq 0$ for all $t \geq T_{2}$. Then $p I(t) \leq Y(t)$ for all $t \geq T_{2}$. It follows from the last equation of system (2.1) that

$$
\frac{\mathrm{d} Y(t)}{\mathrm{d} t}>\frac{1}{p} \gamma(t) X(t) Y(t)-\mu(t) Y(t)=\left(\frac{1}{p} \gamma(t) X(t)-\mu(t)\right) Y(t)
$$

for all $t \geq T_{2}$. Hence, we obtain

$$
Y(t)>Y\left(T_{2}\right) \exp \left(\int_{T_{2}}^{t}\left\{\frac{1}{p} \gamma(s) N_{v}(s)-\mu(s)\right\} \mathrm{d} s\right)
$$

for all $t \geq T_{2}$. From the equality (4.2), we see that there exist constants $\eta_{1}>0$ and $T>0$ such that

$$
\int_{t}^{t+\lambda}\left\{\frac{1}{p} \gamma(s) N_{v}(s)-\mu(s)\right\} \mathrm{d} s>\eta_{1}
$$

for all $t>T$. For convenience, we choose $T_{2}$ satisfying $T_{2} \geq T$. Then the inequality (4.3) holds for $t \geq T_{2}$, it follows from (4.4) that $\lim _{t \rightarrow+\infty} I(t)=+\infty$. This contradicts with the boundedness of $Y(t)$, stated in 2) of Proposition 2.2. Thus we have $W(p, t)>0$ for all $t \geq T_{2}$.

Using Lemma 4.4 we prove Theorem 4.2.

Proof (Proof of Theorem 4.2). For simplicity, let $m_{1 \varepsilon} \triangleq m_{1}-\varepsilon$, $M_{1 \varepsilon} \triangleq M_{1}+\varepsilon, \quad m_{2 \varepsilon} \triangleq m_{2}-\varepsilon$, and $M_{2 \varepsilon} \triangleq M_{2}+\varepsilon$, where $\varepsilon>0$ is a constant. From the inequality (2.7) and (2.8), we see that for any $\varepsilon>0$, there exists $T>0$ such that 


$$
\begin{aligned}
& m_{1} \varepsilon<N(t)<M_{1} \varepsilon, \\
& m_{2} \varepsilon<N_{v}(t)<M_{2} \varepsilon,
\end{aligned}
$$

for all $t \geq T$. The inequality (4.1) and (4.2) implies that for sufficient small $\eta>0$, there exists $T_{1} \geq T$ such that

$$
\begin{gathered}
\int_{t}^{t+\lambda}\left\{\left(\beta_{1}(s)+p \beta_{1}(s)\right) N(s) p-\mu(s)\right\} \mathrm{d} s>\eta, \\
\int_{t}^{t+\lambda}\left\{\frac{1}{p} \gamma(s) N_{v}(s)-\mu_{v}(s)\right\} \mathrm{d} s>\eta
\end{gathered}
$$

for all $t \geq T_{1}$. We define

$$
\begin{aligned}
& \beta_{1}^{+} \triangleq \sup _{t \geq 0} \beta_{1}(t), \beta_{2}^{+} \triangleq \sup _{t \geq 0} \beta_{2}(t), \mu^{+} \triangleq \sup _{t \geq 0} \mu(t), \\
& \mu_{v}^{+} \triangleq \sup _{t \geq 0} \mu_{v}(t), \gamma^{+} \triangleq \sup _{t \geq 0} \gamma(t) .
\end{aligned}
$$

From (4.6) and (4.8), we see that for positive constants $\eta_{1}<\eta$ and $T_{2} \geq T_{1}$ there exist small $\varepsilon_{i}>0,(i=1,2,3,4)$ such that

$$
\begin{gathered}
\int_{t}^{t+\lambda}\left\{\frac{1}{p} \gamma(s)\left(N_{v}(s)-\varepsilon_{1}-\gamma^{+} M_{2 \varepsilon} \omega_{4} \varepsilon_{2}\right)-\mu(s)\right\} \mathrm{d} s>\eta_{1}, \\
N_{v}(t)-\varepsilon_{1}-\gamma^{+} M_{2 \varepsilon} \omega_{4} \varepsilon_{2}>m_{2 \varepsilon},
\end{gathered}
$$

hold for all $t \geq T_{2}$. From 2) of Assumption 2.1, $\varepsilon_{1}, \varepsilon_{2}$ can be chosen sufficiently small satisfying

$$
\int_{t}^{t+\omega_{4}}\left(\gamma(s) M_{2 \varepsilon} \varepsilon_{2}-\mu_{v}(s) \varepsilon_{1}\right) \mathrm{d} s<-\eta_{1},
$$

hold for all $t \geq T_{2}$.

First we claim that $\sup _{t \rightarrow+\infty} I(t)>\varepsilon_{2}$.

In fact, if it is not true, then there exists $T_{3} \geq T_{2}$ such that

$$
I(t) \leq \varepsilon_{2},
$$

for all $t \geq T_{3}$. Suppose that $Y(t) \geq \varepsilon_{1}$ for all $t \geq T_{3}$. Then, from (4.5) and (4.12) we have

$$
\begin{aligned}
Y(t) & =Y\left(T_{3}\right)+\int_{T_{3}}^{t}\left\{\gamma(s) I(s)\left(N_{v}(s)-Y(s)-Y(s)\right)-\mu_{v}(s) Y(s)\right\} \mathrm{d} s \\
& \leq Y\left(T_{3}\right)+\int_{T_{3}}^{t}\left(\gamma(s) M_{2 \varepsilon} \varepsilon_{2}-\mu_{v}(s) \varepsilon_{1}\right) \mathrm{d} s .
\end{aligned}
$$

for all $t \geq T_{3}$. Thus, from (4.11), we have $\lim _{t \rightarrow+\infty} Y(t)=-\infty$, which contradicts with 2) of Proposition 2.2. Therefore we see that there exists $s_{1} \geq T_{3}$ such that $Y\left(s_{1}\right)<\varepsilon_{1}$. Suppose that there exists an $s_{2} \geq s_{1}$ such that $Y\left(s_{2}\right)>\varepsilon_{1}+\gamma^{+} M_{2 \varepsilon} \omega_{4} \varepsilon_{2}$. Then, we see that there necessarily exists an $s_{3} \in\left(s_{1}, s_{2}\right)$ such that $Y\left(s_{3}\right)=\varepsilon_{1}$ and $Y(t)>\varepsilon_{1}$ for all $t \in\left(s_{3}, s_{2}\right]$. Let $n$ be an integer such that $s_{2} \in\left[s_{3}+n \omega_{4}, s_{3}+(n+1) \omega_{4}\right)$. Then from (4.11), we have

$$
\begin{aligned}
& \varepsilon_{1}+\gamma^{+} M_{2 \varepsilon} \omega_{4} \varepsilon_{2} \\
& <Y\left(s_{2}\right)=Y\left(s_{3}\right)+\int_{s_{3}}^{s_{2}}\left\{\gamma(s) I(s)\left(N_{v}(s)-Y(s)\right)-\mu_{v}(s) Y(s)\right\} \mathrm{d} s
\end{aligned}
$$




$$
\begin{aligned}
& <\varepsilon_{1}+\left\{\int_{s_{3}}^{s_{3}+n \omega_{4}}+\int_{s_{3}+n \omega_{4}}^{s_{2}}\right\}\left\{\gamma(s) M_{2 \varepsilon} \varepsilon_{2}-\mu_{v}(s) \varepsilon_{1}\right\} \mathrm{d} s \\
& <\varepsilon_{1}+\int_{s_{3}+n \omega_{4}}^{s_{2}} \gamma(s) M_{2 \varepsilon} \varepsilon_{2} \mathrm{~d} s \\
& <\varepsilon_{1}+\gamma^{+} M_{2 \varepsilon} \omega_{4} \varepsilon_{2}
\end{aligned}
$$

which is a contradiction. Therefore, we see that

$$
Y(t) \leq \varepsilon_{1}+\gamma^{+} M_{2 \varepsilon} \omega_{4} \varepsilon_{2},
$$

for all $t \geq s_{1}$. Now, from lemma 4.4, there exists $T_{4} \geq \tilde{s}_{1}$ such that $W(p, t)>0$ for all $t \geq T_{4}$. Then

$$
\begin{aligned}
\frac{\mathrm{d} Y(t)}{\mathrm{d} t} & =\gamma(t) I(t)\left(N_{v}(t)-Y(t)\right)-\mu_{v}(t) Y(t) \\
& \geq Y(t)\left\{\frac{1}{p} \gamma(t)\left(N_{v}(t)-I_{v}(t)\right)-\mu_{v}(t)\right\} \\
& \geq Y(t)\left\{\frac{1}{p} \gamma(t)\left(N_{v}(t)-\varepsilon_{1}-\gamma^{+} M_{2 \varepsilon} \omega_{4} \varepsilon_{2}\right)-\mu_{v}(t)\right\}
\end{aligned}
$$

for all $t \geq T_{4}$. Hence, we have

$$
Y(t) \geq Y\left(T_{4}\right) \exp \left(\int_{T_{4}}^{t}\left\{\frac{1}{p} \gamma(s)\left(N_{v}(s)-\varepsilon_{1}-\gamma^{+} M_{2 \varepsilon} \omega_{4} \varepsilon_{2}\right)-\mu_{v}(s)\right\} \mathrm{d} s\right)
$$

It follows from (4.9) that $\lim _{t \rightarrow+\infty} Y(t)=+\infty$ and this contradicts with the boundedness of $I_{v}(t)$, stated in 2) of Proposition 2.2. Thus, we see that our claim $\sup _{t \rightarrow+\infty} I(t)>\varepsilon_{2}$ is true.

Next, we prove

$$
\liminf _{t \rightarrow+\infty} I(t) \geq I_{1}
$$

where $I_{1}>0$ is a constant given in the following lines. For the following convenience, we let $\omega$ be the least common multiple of $\omega_{4}$ and $\lambda$. If we define

$$
\liminf _{t \rightarrow+\infty}\left\{\frac{1}{p} \gamma(t)\left(N_{v}(t)-\varepsilon_{1}-\gamma^{+} M_{2 \varepsilon} \omega_{4} \varepsilon_{2}\right)-\mu(t)\right\}:=\underline{m}
$$

Then we have two cases to discuss, namely 1) $\underline{m}>0$ and 2) $\underline{m} \leq 0$. Firstly, we discuss the case 1$)$. We set $\varepsilon>0$ such that $\underline{m}-\varepsilon>0$, then there exist $\widetilde{T_{3}}\left(\geq T_{2}\right)$ such that

$$
\frac{1}{p} \gamma(t)\left(N_{v}(t)-\varepsilon_{1}-\gamma^{+} M_{2 \varepsilon} \omega_{4} \varepsilon_{2}\right)-\mu(t)>\underline{m}-\varepsilon
$$

for all $t \geq \widetilde{T_{3}}$. Then, from inequalities (4.9), (4.11)-(4.12) and 2) of Assumption 2.1 , we see that there exist constants $\widetilde{T}_{4}\left(\geq \widetilde{T}_{3}\right), \lambda_{2}>0$, which is an integral multiple of $\omega$, and $\eta_{2}>0$ such that

$$
\begin{gathered}
\int_{t}^{t+\lambda_{3}}\left\{\gamma(s) M_{2 \varepsilon} \varepsilon_{2}-\mu_{v}(s) \varepsilon_{1}\right\} \mathrm{d} s<-M_{2 \varepsilon}, \\
\int_{t}^{t+\lambda_{3}}\left\{\frac{1}{p} \gamma(s)\left(N_{v}(s)-\varepsilon_{1}-\gamma^{+} M_{2 \varepsilon} \omega_{4} \varepsilon_{2}\right)-\mu(s)\right\} \mathrm{d} s>\eta_{2},
\end{gathered}
$$




$$
\int_{t}^{t+\lambda_{3}} \gamma(s) \mathrm{d} s>\eta_{2},
$$

for all $t \geq \widetilde{T_{4}}$ and $\lambda_{3} \geq \lambda_{2}$. Let $C>0$ be an integer multiple of $\lambda_{2}$ satisfying

$$
e^{-\mu^{+} \lambda_{2}} \eta_{2} m_{2 \varepsilon} v_{2} e^{\frac{C}{\lambda_{2}} \eta_{2}}>\varepsilon_{1}+\gamma^{+} M_{2 \varepsilon} \omega_{4} \varepsilon_{2},
$$

where $v_{2} \triangleq \varepsilon_{2} e^{-2 \mu_{x}^{+} \lambda_{2}}$. Since we have proved $\sup _{t \rightarrow+\infty} I(t)>\varepsilon_{2}$. There are only two possibilities as follows:

1) $I(t) \geq \varepsilon_{2}$ for all $t \geq \exists \widetilde{T_{5}} \geq \widetilde{T_{4}}$.

2) $I(t)$ oscillates about $\varepsilon_{2}$ for large $t \geq \widetilde{T}_{4}$. In case 1 ), we have $\liminf _{t \rightarrow+\infty} I(t) \geq \varepsilon_{2} \triangleq I_{1}$. In case 2 ), there necessarily exist two constants $t_{1}, t_{2} \geq \widetilde{T_{4}}\left(t_{2} \geq t_{1}\right)$ such that

$$
\left\{\begin{array}{l}
I\left(t_{1}\right)=I\left(t_{2}\right)=\varepsilon_{2}, \\
I(t)<\varepsilon_{2}, \text { for all } t \in\left(t_{1}, t_{2}\right) .
\end{array}\right.
$$

Suppose that $t_{2}-t_{1} \leq C+2 \lambda_{2}$. Then, from (1.1) we have

$$
\frac{\mathrm{d} I(t)}{\mathrm{d} t} \geq-\mu^{+} I(t)
$$

Hence, we obtain

$$
I(t) \geq I\left(t_{1}\right) \exp \left(\int_{t_{1}}^{t}-\mu^{+} \mathrm{d} s\right) \geq \varepsilon_{2} e^{-\mu^{+}\left(C+2 \lambda_{2}\right)}:=I_{1},
$$

for all $t \in\left(t_{1}, t_{2}\right)$. Suppose that $t_{2}-t_{1}>C+2 \lambda_{2}$. Then, from (4.18), we have

$$
I(t) \geq \varepsilon_{2} e^{-\mu^{+}\left(C+2 \lambda_{2}\right)}=I_{1}
$$

for all $t \in\left(t_{1}, t_{1}+C+2 \lambda_{2}\right)$. Now, we are in a position to show that $I(t) \geq I_{1}$ for all $t \in\left[t_{1}+C+2 \lambda_{2}, t_{2}\right)$. Suppose that $I(t) \geq \varepsilon_{1}$ for all $t \in\left[t_{1}, t_{1}+2 \lambda_{2}\right]$. Then, from (4.14), we have

$$
Y\left(t_{1}+\lambda_{2}\right) \leq Y\left(t_{1}\right)+\int_{t_{1}}^{t_{1}+\lambda_{2}}\left\{\gamma(s) M_{2 \varepsilon} \varepsilon_{2}-\mu_{v}(s) \varepsilon_{1}\right\} \mathrm{d} s<M_{2 \varepsilon}-M_{2 \varepsilon}=0
$$

which is a contradiction. Therefore, there exists an $s_{4} \in\left[t_{1}, t_{1}+2 \lambda_{2}\right]$ such that $Y\left(s_{4}\right)<\varepsilon_{1}$. Then, as is in the proof of $\sup _{t \rightarrow+\infty} I(t)>\varepsilon_{2}$, we can show that

$$
Y(t) \leq \varepsilon_{1}+\gamma^{+} M_{2 \varepsilon} \omega_{4} \varepsilon_{2},
$$

for all $t \geq s_{4}$. From (4.18), we have

$$
I(t) \geq v_{2}=\varepsilon_{2} e^{-2 \mu^{+} \lambda_{2}},
$$

for all $t \in\left[t_{1}, t_{1}+2 \lambda_{2}\right]$. Thus, from (4.10), (4.20), (4.21), we have

$$
\frac{\mathrm{d} Y(t)}{\mathrm{d} t}=\gamma(t) I(t) X(t)-\mu_{v}(t) Y(t) \geq \gamma(t) m_{2 \varepsilon} v_{2}-\mu_{v}^{+} Y(t)
$$

for all $t \in\left[t_{1}+\lambda_{2}, t_{1}+2 \lambda_{2}\right]$. Hence, from (4.16), we obtain

$$
\begin{aligned}
& Y\left(t_{1}+2 \lambda_{2}\right) \\
& \geq e^{-\mu^{+}\left(t_{1}+2 \lambda_{2}\right)}\left\{Y\left(t_{1}+\lambda_{2}\right) e^{\mu^{+}\left(t_{1}+\lambda_{2}\right)}+\int_{t_{1}+\lambda_{2}}^{t_{1}+2 \lambda_{2}} \gamma(s) m_{2 \varepsilon} v_{2} e^{\mu^{+} s} \mathrm{~d} s\right\} \\
& \geq e^{-\mu^{+}\left(t_{1}+2 \lambda_{2}\right)} \int_{t_{1}+\lambda_{2}}^{t_{1}+2 \lambda_{2}} \gamma(s) m_{2 \varepsilon} v_{2} e^{\mu^{+} s} \mathrm{~d} s \geq e^{-\mu^{+} \lambda_{2}} \eta_{2} m_{2 \varepsilon} v_{2} .
\end{aligned}
$$


Now we suppose that there exists a $t_{0}>0$ such that $t_{0} \in\left(t_{1}+2 \lambda_{2}+C, t_{2}\right)$, $I\left(t_{0}\right)=I_{1}$ and $I(t) \geq I_{1}$ for all $t \in\left[t_{1}, t_{0}\right]$. Then there exists $m \in N$ such that $t_{0} \in\left[t_{1}+2 \lambda_{2}+C+m \omega, t_{1}+2 \lambda_{2}+C+(m+1) \omega\right)$. Note that from Lemma 4.4.

without loss of generality, we can assume that $t_{1}$ is so large that $W(p, t)=p I(t)-Y(t)>0$ for all $t \geq t_{1}+2 \lambda_{2}$. Then, from (4.20), we have

$$
\begin{aligned}
\frac{\mathrm{d} Y(t)}{\mathrm{d} t} & =\gamma(t)\left(N_{v}(t)-Y(t)\right) I(t)-\mu_{v}(t) Y(t) \\
& \geq Y(t)\left\{\frac{1}{p} \gamma(t)\left(N_{v}(t)-Y\right)(t)-\mu_{v}(t)\right\} \\
& \geq Y(t)\left\{\frac{1}{p} \gamma(t)\left(N_{v}(t)-\varepsilon_{1}-\gamma^{+} M_{2 \varepsilon} \omega_{4} \varepsilon_{2}\right)-\mu_{v}(t)\right\}
\end{aligned}
$$

for all $t \in\left(t_{1}+2 \lambda_{2}, t_{2}\right)$. Thus, from (4.15) and (4.22), we have

$$
\begin{aligned}
Y\left(t_{0}\right) \geq & Y\left(t_{1}+2 \lambda_{2}\right) \exp \left(\int_{t_{1}+2 \lambda_{2}}^{t_{0}}\left\{\frac{1}{p} \gamma(t)\left(N_{v}(s)-\varepsilon_{1}-\gamma^{+} M_{2 \varepsilon} \omega_{4} \varepsilon_{2}\right)-\mu_{v}(s)\right\} \mathrm{d} s\right) \\
\geq & e^{-\mu^{+} \lambda_{2}} \eta_{2} m_{2 \varepsilon} v_{2} \exp \left(\left\{\int_{t_{1}+2 \lambda_{2}}^{t_{1}+2 \lambda_{2}+C}+\int_{t_{1}+2 \lambda_{2}}^{t_{1}+2 \lambda_{2}+C+m \omega}+\int_{t_{1}+2 \lambda_{2}+C+m \omega}^{t_{0}}\right\}\right. \\
& \left.\left\{\frac{1}{p} \gamma(t)\left(N_{v}(s)-\varepsilon_{1}-\gamma^{+} M_{2 \varepsilon} \omega_{4} \varepsilon_{2}\right)-\mu_{v}(s)\right\} \mathrm{d} s\right) \\
\geq & e^{-\mu^{+} \lambda_{2}} \eta_{2} m_{2 \varepsilon} v_{2} e^{\frac{C}{\lambda_{2}} \eta_{2}}
\end{aligned}
$$

Thus, from (4.20), we have

$$
\varepsilon_{1}+\gamma^{+} M_{2 \varepsilon} \omega_{4} \varepsilon_{2} \geq e^{-\mu^{+} \lambda_{2}} \eta_{2} m_{2 \varepsilon} v_{2} e^{\frac{C}{\lambda_{2}} \eta_{2}},
$$

which contradicts with (4.17). Finally, if $\underline{m} \leq 0$, we let $C>0$ be the integral multiple of $\lambda_{2}$ satisfying

$$
e^{-\mu_{1}^{+} \lambda_{2}} \eta_{2} m_{2 \varepsilon} \nu_{2} e^{\frac{C}{\lambda_{2}} \eta_{2}+(\underline{m}-\varepsilon) \omega}>\varepsilon_{1}+\gamma^{+} M_{2 \varepsilon} \omega_{4} \varepsilon_{2},
$$

Then, repeating the above steps, we have

$$
Y\left(t_{0}\right) \geq e^{-\mu^{+} \lambda_{2}} \eta_{2} m_{2 \varepsilon} v_{2} e^{\frac{C}{\lambda_{2}} \eta_{2}+(\underline{m}-\varepsilon) \omega}
$$

Thus, from (4.20), we have

$$
\varepsilon_{1}+\gamma^{+} M_{2 \varepsilon} \omega_{4} \varepsilon_{2} \geq e^{-\mu^{+} \lambda_{2}} \eta_{2} m_{2 \varepsilon} \nu_{2} e^{\frac{C}{\lambda_{2}} \eta_{2}+(\underline{m}-\varepsilon) \omega}
$$

which is contradictive with (4.23). Therefore, $I(t) \geq I_{1}$ for all $t \in\left[t_{1}+2 \lambda_{2}+C, t_{2}\right)$, which implies $\liminf _{t \rightarrow+\infty} I(t) \geq I_{1}$.

Since $\limsup _{t \rightarrow+\infty} I(t) \leq \limsup _{t \rightarrow+\infty} N(t) \leq M_{1}<+\infty$, the infectious population of system (1.1) is permanent.

\section{Discussion}

In the paper, we have extended the epidemic models of vector-borne disease with direct mode of transmission presented in [20]. A non-autonomous vector 
infectious disease model that conforms to the actual environment has been established, which combines the spread of epidemics with changes in the natural environment and fully reflects the characteristics of the spread of epidemics that change over time. There are relatively few popular articles on the establishment of non-autonomous mathematical models, so the non-autonomous vector infectious disease models are even rarer. Therefore, our research has a certain theoretical value and application value.

\section{Acknowledgements}

The work was supported by the Science Fund of Education Department of Jiangxi Province $(171373,171374,181361)$.

\section{Conflicts of Interest}

The authors declare no conflicts of interest regarding the publication of this paper.

\section{References}

[1] Peiris, J., Chu, C.M., Cheng, V.C.C., Chan, K.S., Hung, I.F.N., Poon, L.L.M., et al. (2003) Clinical Progression and Viral Load in a Community Outbreak of Coronavirus-Associated SARS Pneumonia: A Prospective Study. Lancet, 361, 1767-1772. https://doi.org/10.1016/S0140-6736(03)13412-5

[2] Novel Swine-Origin Influenza A (H1N1) Virus Investigation Team (2009) Emergence of a Novel Swine-Origin Influenza A (H1N1) Virus in Humans. The New England Journal of Medicine, 360, 2605-2615.

https://doi.org/10.1056/NEJMoa0903810

[3] Jia, J.W. and Qin, G.L. (2017) Stability Analysis of HIV/AIDS Epidemic Model with Nonlinear Incidence and Treatment. Advances in Difference Equations, 2107, Article No. 136. https://doi.org/10.1186/s13662-017-1175-5

[4] Wang, X.Y., Liu, X.Z., Xie, W.C., Xu, W. and Xu, W. (2016) Global Stability and Persistence of HIV Models with Switching Parameters and Pulse Control. Mathematics and Computers in Simulation, 123, 53-67. https://doi.org/10.1016/j.matcom.2015.12.008

[5] Gao, S.J., Teng, Z.D., Nieto, J.J. and Torres, A. (2007) Analysis of an SIR Epidemic Model with Pulse Vaccination and Distributed Time Delay. Journal of Biomedicine and Biotechnology, 2007, Article ID: 064870. https://doi.org/10.1155/2007/64870

[6] Wang, J.J., Zhang, J.Z. and Jin, Z. (2010) Analysis of an SIR Model with Bilinear Incidence Rate. Nonlinear Analysis: Real World Applications, 11, 2390-2402. https://doi.org/10.1016/j.nonrwa.2009.07.012

[7] Liu, X. and Peter, S. (2017) Switching Vaccination Schemes for Vector-Borne Diseases with Seasonal Fluctuations. Journal of Biological Systems, 25, 441-477. https://doi.org/10.1142/S0218339017500218

[8] Xue, Y. and Wang, J. (2012) Backward Bifurcation of an Epidemic Model with Infectious Force in Infected and Immune Period and Treatment. Abstract and Applied Analysis, 2012, Article ID: 647853. https://doi.org/10.1155/2012/647853

[9] Hussaini, N. and Winter, M. (2010) Travelling Waves for an Epidemic Model with Non-Smooth Treatment Rates. Journal of Statistical Mechanics, 2010, 11019. 
https://doi.org/10.1088/1742-5468/2010/11/P11019

[10] Brauer, F. (2008) Epidemic Models with Heterogeneous Mixing and Treatment. Bulletin of Mathematical Biology, 70, Article No. 1869.

https://doi.org/10.1007/s11538-008-9326-1

[11] Hu, Z.X., Liu, S. and Wang, H. (2008) Backward Bifurcation of an Epidemic Model with Standard Incidence Rate and Treatment Rate. Nonlinear Analysis. Real World Applications, 9, 2302-2312. https://doi.org/10.1016/j.nonrwa.2007.08.009

[12] Wang, W.D. and Ruan, S.G. (2004) Bifurcations in an Epidemic Model with Constant Removal Rate of the Infectives. Journal of Mathematical Analysis and Applications, 291, 775-793. https://doi.org/10.1016/j.jmaa.2003.11.043

[13] Wang, W.D. (2006) Backward Bifurcation of an Epidemic Model with Treatment. Mathematical Biosciences, 201, 58-71. https://doi.org/10.1016/j.mbs.2005.12.022

[14] Guo, Z.Y., Huang, L.H. and Zou, X.F. (2012) Impact of Discontinuous Treatments on Disease Dynamics in an SIR Epidemic Model. Mathematical Biosciences and Engineering, 9, 97-110. https://doi.org/10.3934/mbe.2012.9.97

[15] Zhang, T.L., Kang, R.N., Wang, K. and Liu, J.L. (2015) Global Dynamics of an SEIR Epidemic Model with Discontinuous Treatment. Advances in Difference Equations, 2015, Article No. 136. https://doi.org/10.1186/s13662-015-0695-0

[16] Zhang, Z.H. and Suo, Y.H. (2010) Qualitative Analysis of a SIR Epidemic Model with Saturated Treatment Rate. Journal of Applied Mathematics and Computing, 34, 177-194. https://doi.org/10.1007/s12190-009-0315-9

[17] Zhang, X. and Liu, X.N. (2008) Backward Bifurcation of an Epidemic Model with Saturated Treatment Function. Journal of Mathematical Analysis and Applications, 348, 433-443. https://doi.org/10.1016/j.jmaa.2008.07.042

[18] Cosner, C., Beier, J.C., Cantrell, R.S., Impoinvil, D., Kapitanski, L., Potts, M.D., et al. (2009) The Effects of Human Movement on the Persistence of Vector-Borne Diseases. Journal of Theoretical Biology, 258, 550-560.

https://doi.org/10.1016/j.jtbi.2009.02.016

[19] Hossain, S., Nayeem, J. and Podder, C. (2015) Effective Control Strategies on the Transmission Dynamics of a Vector-Borne Disease. Open Journal of Modelling and Simulation, 3, 111-119. http://dx.doi.org/10.4236/ojmsi.2015.33012

[20] Shi, R.Q., Zhao, H.Y. and Tang, S.Y. (2014) Global Dynamic Analysis of a Vector-Borne Plant Disease Model. Advances in Difference Equations, 2014, Article No. 59. https://doi.org/10.1186/1687-1847-2014-59 